\title{
Review of recently approved glaucoma surgical innovations
}

\begin{abstract}
Cataracts and glaucoma are the two most common causes of blindness worldwide. Since the 1800 's ophthalmologists have been working to improve various approaches for reducing the elevated intraocular pressure (IOP) that occurs in both angle closure and open angle glaucoma $(\mathrm{OAG})$. A recent trend has emerged referred to as minimally invasive glaucoma surgery (MIGS) procedures that share the common goal of reducing complications, and ocular morbidity associated with traditional incisional surgical management of glaucoma while maintaining a similar efficacy. Three new products or "microstents" have recently been approved by the FDA: iStent, CyPass, and Xen Implant for the management of chronic open angle glaucoma in patients who are refractory to other forms of management including pharmacotherapy and laser trabeculoplasty. Prior to FDA approval, several studies compared the efficacy of concurrent cataract surgery and microstent implantation to that of phacoemulsification alone. The Cypass and iStent were approved after demonstrating a reduction in IOP and topical IOP lowering therapy versus cataract surgery alone. Studies so far have compared the rates of IOP lowering, adverse events, and ocular hypotensive medication use at the end of a 12 month or 24-month follow-up periods. With the exception of the Xen implant, as published data outside of the FDA approval is forthcoming and the implant was placed in the absence of a concurrent cataract surgery, both the iStent and Cypass devices appear to have superior IOP lowering effect yet similar adverse events to a standard cataract surgery in isolation. This article summarizes the data of the major studies leading to the FDA approval of these devices.
\end{abstract}

Keywords: glaucoma, minimally invasive glaucoma surgery, istent, cypass, xen implant, microstent
Volume 6 Issue 6 - 2017

Mark P Grogan, Shane J Havens

University of Nebraska Medical Center, USA

Correspondence: MP Grogan, University of Nebraska Medical Center, 985540 Nebraska Medical Center, USA, Tel 4023204329, Emailshavens@unmc.edu

Received: March 23, 2017 | Published: April 21, 2017
Abbreviations: MIGS, minimally invasive glaucoma surgery; IOP, intraocular pressure; POAG, primary open angle glaucoma; $\mathrm{OAG}$, open angle glaucoma

\section{Introduction}

After cataract, glaucoma is the second most prevalent cause of blindness worldwide. ${ }^{1}$ There are two major types of Glaucoma contributing to this worldwide problem, open angle and closed angle glaucoma. OAG is a slowly progressive process, primarily affecting adults. It results in optic nerve atrophy and cupping in addition to retinal damage over the course of years, with the majority remaining asymptomatic until advanced stages. ${ }^{2}$ Angle closure glaucoma on the other hand, results from acute closure of the anterior chamber angle resulting in a rapid increase in IOP, with subsequent optic nerve and ganglion cell damage. ${ }^{3}$ It is estimated that by 2040 the prevalence of glaucoma of both types will increase from approximately 64 million in 2013 to nearly 111 million. ${ }^{4}$ Major risk factors associated with acute angle closure glaucoma include age, female gender, family history, and Asian or Inuit descent. Signs associated with an increased risk of acute angle closure glaucoma include shallow peripheral and/ or central anterior chamber depth, thick crystalline lenses, and short axial length of the eye, ciliary body configuration, hyperopia, and steep corneal curvature. ${ }^{3}$

Risk factors for the development of OAG include age, family history in a first degree relative, African descent, Signs associated with an elevated risk of progression to OAG include elevated IOP, a thinner central cornea, lower intraocular perfusion pressure, disc hemorrhage, and a larger cup to disc ratio. ${ }^{2}$ The approach to management of patients with glaucoma differs based on type, open vs. closed. Acute angle closure glaucoma management is considered an ophthalmic emergency and treatment is aimed at lowering the IOP in as soon as possible. This can be accomplished with a combination of pharmacologic and/or surgical interventions, of which laser iridotomy is the preferred approach. ${ }^{3}$ Approaches to the management of OAG include a combination of medications, laser trabeculoplasty and/or other surgical methods. ${ }^{2}$ Ideas encompassing surgical management of glaucoma have been around since the mid1800. During this time, German ophthalmologist Albrecht von Graefe began to utilize iridectomy as a means of lowering IOP. Not long after, French ophthalmologist, Louis de Wecker designed a procedure to facilitate efflux of aqueous humor from the anterior chamber to the subconjunctival space. In 1876, he was successful in utilizing a golden conduit to create the first known implantable shunt in glaucoma patient's refractory to iridectomy. ${ }^{5}$ In recent years, there have been efforts to develop novel surgical devices to improve outcomes, and reduce the risk and optimize vision recovery following glaucoma surgery, along with efforts to avoid the creation of a conjunctival bleb. The conjunctival bleb can leak and poses risk of late infectious complications that carry great ocular morbidity. ${ }^{6}$ Following studies demonstrating the ability of cataract surgery to lower IOP in those with glaucoma by groups such as Mansberger et $\mathrm{al}^{7}{ }^{\text {it }}$ was hypothesized that the addition of microstent implantation to patients undergoing cataract surgery would have an even greater 
impact on IOP. Three approaches to the management of OAG (iStent, Cypass and the Xen Implant devices) have been developed within the last decade under the category of microinvasive glaucoma surgery (MIGS). All three involve the surgical implantation of microscopic stents, most often while the patient is undergoing cataract surgery. They have all recently received FDA approval for use in the United States. ${ }^{8-10}$ The iStent and Cypass FDA approval label is for combined cataract and iStent or Cypass placement while the Xen implant approval includes a combined procedure or as a stand-alone implant.

\section{Discussion}

A search on clinicaltrials.gov for "iStent", "CyPass", and "Xen Implant" yields 31 studies for iStent, 6 for CyPass, and 3 for Xen Implant respectively. ${ }^{11}$ The $1 \mathrm{~mm}$ long Titanium snorkel shaped iStent, manufactured by Glaukos Corporation in California, works by creating a conduit between the anterior chamber and the canal of Schlemm, effectively bypassing the trabecular meshwork and leading to a potential increase in aqueous humor outflow in OAG. ${ }^{8}$ In 2007 , an original study, analyzing the effect of ocular hypotensive agents on IOP, was conducted by Spiegel, Wetzel, Haffner, and Hill. This prospective case series followed 6 patients with open-angle glaucoma and reported that both a reduction in IOP and a decreased need for glaucoma medications were significant through 1 year following the procedures. Mean IOP prior to implantation of $20.2+/-6.3$ with a decline to a mean of $14-15 \mathrm{mmHg}$ through at least 1 year. ${ }^{12}$ Despite demonstrating the iStent's capacity to lower IOP alone, the studies leading to the FDA approval of this device, in June of 2012, were based on comparisons of iStent implantation alongside phacoemulsification versus cataract surgery alone. ${ }^{8}$ A prospective, randomized, controlled, multi-center clinical trial was conducted by Samuelson, Katz, Wells, Duh, Giamporcaro in 2011, to assess the benefit of implantation of the iStent device at the same time as phacoemulsification cataract surgery, compared with controls who underwent cataract surgery alone. Treatment and control groups were compared in terms of IOP $\leq 21$ $\mathrm{mmHg}$ in patients without medication, 12 months following the procedure. By the end of the study it was determined that the addition of iStent placement to cataract surgery, had a favorable outcome profile, and was a less invasive procedure compared to traditional glaucoma surgeries, like trabeculectomy and glaucoma tube shunt placement. $72 \%$ of patients in the treatment arm were able to show IOP reductions to values equal to or below $21 \mathrm{mmHg}$ without use of intraocular hypotensive medications by 1 year following surgery. In contrast, $50 \%$ met this criterion in the control arm. Additionally, a reduction in IOP by $\geq 20 \%$ in comparison to baseline IOP was noted in $66 \%$ of treatment and $48 \%$ of control group patients. The majority of the adverse events experienced were those commonly seen following phacoemulsification cataract surgery, very similar in rate between the control and experimental arms. Mean declines in IOP at the 12 month follow-up visit were similar between the control and experimental group at $-8.5+/-4.3$ and $8.4+/-3.6$ respectively. ${ }^{1}$

A smaller trial conducted in Italy in 2009 by Fea, Consolandi, Zola, Pignata, Cannizzo et al., also found that long term pressure control was greater in patients who received both cataract surgery and simultaneous iStent implantation. Another recently approved device, the CyPassmicrostent developed by Transcend Medical Inc., United States of America, is a novel microscopic implantable stent aimed at treating glaucoma, through increasing outflow of the aqueous humor through the uveoscleral route, differing from the mechanism of the iStent. It was approved for use by the FDA in July of 2016 for implantation concurrent with cataract surgery in patients suffering from both cataract and glaucoma..$^{10}$ Approval of this device was largely based on a clinical trial conducted by Vold, Ahmed, Craven, Mattox, Stamper et al. ${ }^{13}$ A total of 505 eyes of 505 subjects, were assigned to a treatment group (374 participants), involving a combination of cataract surgery and Cypass implantation, and a control group (131 participants), involving cataract surgery only. Comparison between treatment and control arms was made using the primary objective, the percentage of eyes showing a $\geq 20 \%$ decline in IOP two years out from surgery. Additional measures of efficacy included: comparisons of the fraction of participants with an IOP between 6 and $18 \mathrm{mmHg}$, mean change in IOP, mean ocular hypotensive medication use at the end of the trial, and ocular adverse events. $60 \%$ of controls and $77 \%$ of Cypass recipients achieved their primary efficacy measure. Additionally, $85 \%$ of patients receiving a microstent implant did not require the use of any ocular hypotensive medications at the 24 month goal, compared to $59 \%$ in the control group. Mean decline in IOP at 24 months was $7.4+/-4.4$ for the experimental group, and $5.4+/$ - 3.8 for the control group. Mean ocular hypotensive medication use was 0.2 $+/-0.6$ for the experimental group through 24 months while the control group was at $0.7+/-0.9$ at 12 months and $0.6+/-0.8$ at 24 months. Both the mean decline in IOP at 24 months and mean medication use was deemed statistically significant by the study group. ${ }^{14}$ The most recent FDA approval is the Xen Implant from AqueSys, approved for use in the United States in the fall of 2016, is not slated to begin circulation in this country until sometime in 2017. This device has been approved for, "management of refractory glaucomas, including cases where previous surgical treatment has failed, cases of POAG, and pseudoexfoliative or pigmentary glaucoma with open angles that are unresponsive to maximum tolerated medical therapy". ${ }^{10}$ There has not been much data published regarding this device as it is new to the market in the United States.

The American Academy of Ophthalmology asserts that Rohit Varma, M.D. of the University of Southern California's Roski Eye Institute led a trial showing a decrease in both IOP and number of intraocular hypotensive medications used at 12 months following Xen Implant surgery, leading to its FDA approval in November of $2016 .{ }^{15}$ According to clinicaltrials.gov, there are two ongoing trials assessing the utility of the Xen implant in the management of POAG. One of these studies does not include participants from the United States, and is comparing group patients with POAG receiving the Xen implant alone, to a group receiving simultaneous Xen implant and phacoemulsification. The other study is being carried out in the United States, and is a prospective, multicenter trial is following a single group, all with POAG, receiving the Xen implant alone. ${ }^{11}$ An additional study by Galal, Bilgic, Eltanamly, Osman (2017), has assessed the effectiveness of the Xen implant with concurrent subconjunctival mitomycin C in lowering IOP. 13 eyes were evaluated, 3 of which had already undergone phacoemulsification and an additional 10 underwent simultaneous phacoemulsification with stent placdement. Results were significant for a decline in IOP following stent implantation with a pre-op mean IOP was $16+/-4 \mathrm{mmHg}$ which dropped to $9+/-5 \mathrm{mmHg}$ by 1 week following the implantation and remained decreased at $12+/-3 \mathrm{mmHg}$ at 1 year. ${ }^{16}$

Both the iStent and CyPass FDA approval studies compare the outcomes of treatment of patients with OAG with a combination of microstent placement and cataract surgery to a control group receiving cataract surgery alone. Both studies demonstrate fewer adverse outcomes compared to traditional glaucoma surgery. One adverse 
outcome common to both devices is postoperative stent obstruction. iStent showed a $3.6 \%$ (4/111) obstruction rate compared to the Cypass study demonstrating a rate of only $2.1 \%$. An additional shared adverse outcome between the iStent and Cypass is subconjunctival hemorrhage. In the Samuelson, Katz, Wells, Duh, Giamporcaro study from 2011, both the control and treatment arms shared a subconjunctival hemorrhage rate of $2 \%$. The treatment group in the Vold, Ahmed, Craven, Mattox, Stamper et al. study, showed a 1.6\% rate of subconjunctival hemorrhage with a rate of 0.8 in the control group. Although the CyPass trial observed patients for a longer period of time following the experimental procedure, both demonstrated outcomes favoring the addition of the microstents to cataract surgery as methods of additional IOP lowering. There are no studies in the literature currently that directly compare the MIGS procedures to one another. The greatest theoretical advantage of the iStent and Cypass is the lack of conjunctival manipulation and dissection, avoiding the creation of a conjunctival bleb and its inherent risks of late leak and even infection. ${ }^{1,10}$

\section{Conclusion}

All three devices offer alternatives to traditional surgical treatment of patients with OAG. These additional options allow glaucoma surgeons to tailor the surgical risk and benefit profile to the individual patient based on the severity of their glaucoma, their lifestyle, and target IOP level. Furthermore, these devices hold the potential for a more rapid and meaningful vision recovery following glaucoma surgery. In patients who have trouble with administration of or compliance with topical medication, a combined cataract MIGS surgery can prove to be the safest method of decreasing their need for drops and improving their quality of life. Additionally, compared to traditional glaucoma surgery, these three approaches offer a less invasive method of lowering intra-ocular pressures with an improved safety profile and a more rapid recovery of vision. It will be interesting to discover what the future might hold for these and other novel approaches to the management of glaucoma.

\section{Acknowledgments}

None.

\section{Conflicts of interest}

The author declares that there is no conflict of interest.

\section{References}

1. Samuelson TW, Katz LJ, Wells JM, et al. Randomized evaluation of the trabecular micro-bypass stent with phacoemulsification in patients with glaucoma and cataract. Ophthalmology. 2011;118(3):459-467.
2. Prum BE, Rosenberg LF, Gedde SJ, et al. Primary open-angle glaucoma preferred practice pattern $((\mathrm{R}))$ guidelines.Ophthalmology. 2016;123(1):P41-P111.

3. Prum BE, Herndon LW, Moroi SE, et al. Primary angle closure preferred practice pattern((R)) guidelines. Ophthalmology. 2016;123(1): P1-P40.

4. Tham YC, Li X, Wong TY, et al. Global prevalence of glaucoma and projections of glaucoma burden through 2040: A systematic review and meta-analysis.Ophthalmology. 2014;121(11):2081-2090.

5. Razeghinejad MR, Spaeth GL. A history of the surgical management of glaucoma. Optom Vis Sci. 2011;88(1):E39-47.

6. DeBry PW, Perkins TW, Heatley G, et al. Incidence of late-onset blebrelated complications following trabeculectomy with mitomycin. Arch Ophthalmol. 2002;120(3):297-300.

7. Mansberger SL, Gordon MO, Jampel H, et al. Reduction in intraocular pressure after cataract extraction: The ocular hypertension treatment study. Ophthalmology. 2012;119(9):1826-1831.

8. U.S. Food and Drug Administration. Center for Drug Evaluation and Research.Glaukos iStent@ TrabecularMicro-Bypass Stent (Models: GTS-100R, GTS-100L) andinserter (GTS- I 00i) approvalletter. June 25, 2012.

9. U.S. Food and Drug Administration. Center for Drug Evaluation and Research. CyPass ${ }^{\circledR}$ System, Model 241-S approvalletter. July 29, 2016.

10. U.S. Food and Drug Administration. Center for Drug Evaluation and Research. XenGlaucoma Treatment System approvalletter. October 11, 2016.

11. National Library of Medicine (NLM). A service of the U.S. national institutes of health. 2017.

12. Detlev Spiegel, Wolfgang Wetzel, David S Haffner, et al. Initial clinical experience with the trabecular micro-bypass stent in patients with glaucoma. Advances in Therapy. 2007;24(1):161-170.

13. Fea AM, Consolandi G, Zola M, et al. Micro-bypass implantation for primary open-angle glaucoma combined with phacoemulsification: 4year follow-up. Journal of Ophthalmology. 2015:795357.

14. Vold S, Ahmed, Craven ER, et al. Two-year COMPASS trial results: Supraciliary microstenting with phacoemulsification in patients with openangle glaucoma and cataracts. Ophthalmology. 2016;123(10):2103-2112.

15. American Academy of Ophthalmology. FDA approves xengel stent for glaucoma. 2016.

16. Galal A, Bilgic A, Eltanamly R, et al. XEN glaucoma implant with mitomycin C 1-year follow-up: Result and complications. Journal of Ophthalmology. 2017(2017):5457246. 